\title{
SOME LESSONS LEARNED FROM PUBLIC HEALTH ON THE PROCESS OF ADAPTATION ${ }^{\dagger}$
}

\author{
KRISTIE L. EBI ${ }^{1, *}$, JOEL SMITH ${ }^{2}$, IAN BURTON ${ }^{3}$ and JOEL SCHERAGA ${ }^{4}$ \\ ${ }^{1}$ Exponent Health Group, 1800 Diagonal Road, Suite 300, Alexandria, VA 22314 \\ ${ }^{2}$ Stratus Consulting, 1881 Ninth Street, Suite 201, Boulder, CO 80302 \\ ${ }^{3}$ Scientist Emeritus, Meteorological Service of Canada, Toronto, Canada \\ ${ }^{4}$ Global Change Research Program, EPA, Office of Research \& Development, Mail Code 8601-N, \\ 1200 Pennsylvania Ave. NW, Washington, DC 20460 \\ ('Author for correspondence: Tel: +571-227-7250, Fax: 571-227-7299; \\ E-mail:kebi@exponent.com)
}

(Received 20 July 2004; accepted in final form 4 March 2005)

\begin{abstract}
Lessons learned from more than 150 years of public health research and intervention can provide insights to guide public health professionals and institutions as they design and implement specific strategies, policies, and measures to increase resilience to climate variability and change. This paper identifies both some modifications to public health systems that may enhance adaptive capacity, and lessons drawn from the history of managing environmental and other threats in the public health sector that may have relevance for other sectors as they design approaches to increase their adaptive capacity to more effectively cope with climate variability and change.
\end{abstract}

Keywords: adaptation, adaptive capacity, climate change, impacts, public health

\section{Introduction}

Industrial civilization, with its heavy reliance on fossil fuels, is changing the climate because the emission of greenhouse gases due to human activities enhances the natural greenhouse effect (Watson et al. 2001). Climate change has the potential to affect multiple sectors with different intensity across regions and time, such as altering the frequency of occurrence and range of climate-sensitive diseases. It is a complex problem for policymakers to address because it involves interrelationships among physical, social, economic, political, and other factors, and because it is surrounded by significant scientific uncertainties (Watson et al. 2001). However, the increasing understanding of the ways that weather and climate variability are likely to change over the coming decades offers an opportunity to proactively design and implement adaptation strategies, policies, and measures to reduce projected negative impacts and take advantage of new opportunities. The effectiveness of adaptation will determine the degree to which individuals and communities will experience health effects due to climate change (Smit et al. 2001). Failure to invest in adaptation may leave a nation poorly prepared to cope with adverse changes and increase the probability of severe consequences (Smith and Lenhart 1996).

\footnotetext{
${ }^{\dagger}$ The views expressed are the author's own and do not represent official US EPA policy.
} 
Human societies have experience with adaptation to climate-related risks, having responded with varying degrees of success to climate variability and extreme events. It is a testament to human ingenuity and adaptability that societies have been established and flourished in a wide range of climatic environments, given sufficient resources. However, as our understanding of the sensitivity of human health and other systems to climate change has increased, so has our awareness of the challenges posed by ongoing climate change (McMichael 2001).

Working Group II of the Intergovernmental Panel on Climate Change (IPCC) concluded in their Third Assessment Report that advances had been made since previous IPCC assessments in the detection of change in biotic and physical systems, and steps had been taken to improve the understanding of adaptive capacity, vulnerability to climate extremes, and other critical impact-related issues (Smit et al. 2001). These advances indicate a need for initiatives to begin designing adaptation strategies and building adaptive capacities, the underlying goal of which is the protection and enhancement of human health and well-being.

Public health has a long history of effectively intervening to reduce risks to the health of individuals and communities. Lessons learned from more than 150 years of research and intervention can provide insights to guide the design and implementation of effective and efficient strategies, policies, and measures to reduce the potential negative impacts of and to take advantage of the opportunities of climate variability and change. Public health professionals have a wealth of experience in responding to both gradual long-term changes (disease rates changing over decades) and stimuli with severe consequences across short time scales (disease outbreaks). In addition, some intervention programs have been in place for decades. Numerous examples exist of the efficacy and effectiveness of intervention programs, as do examples that demonstrate limits to the resilience of public health systems (Ebi et al. in press). As such, public health offers a wealth of case studies that illustrate how a particular system responded to a specific stress, whether on a small or large scale.

The following lessons are relevant both to public health and to other sectors when considering approaches to decrease the vulnerability and increase the adaptive capacity of individuals and communities. Understanding how these lessons were learned and their consequences can provide guidance when designing and implementing strategies, policies, and measures to address current and projected impacts of climate variability and change.

\section{Lessons Learned}

The lessons can be grouped into the following five themes (Burton et al. in press):

1. Adaptation is a process that requires sustained commitment. Continuous monitoring and regular evaluation of interventions are needed because health risks and their drivers change over time. It is prudent to intervene on a small scale to test the effectiveness of solutions before large-scale deployment. 
2. An understanding of the multiple and interacting determinants of disease is needed to inform the design of adaptation strategies, policies, and measures. Climate change may exacerbate or ameliorate disease determinants, with the possibility that thresholds, nonlinearities, or surprises may be encountered. Increased understanding of the health impacts of current climate variability is likely to facilitate adaptation to future climatic conditions.

3. Multiple political, social, economic, technological, and human factors determine whether adaptation strategies, policies, and measures are effective. Therefore, interventions embedded in an understanding of human factors and tailored to address local situations are likely to be more effective. Also, maladaptation and unintended consequences of interventions can occur in many different ways.

4. Surveillance and early warning systems, coupled with effective response capabilities, can reduce current and future vulnerability.

5. Collaboration and coordination of research and response are required across sectors.

An underlying theme is the need to establish an institutional structure to maintain vigilance in responding to climate variability and change, and to commit sufficient resources on an ongoing basis for the identification of and response to problems. An effective institutional structure can facilitate the "mainstreaming" of the projected impacts of climate change into the design of adaptation interventions in public health and other sectors.

Effective interventions rarely result from a one-off intervention. The aphorism of acting in haste and repenting at leisure applies, with the caveat that the consequences of a less than effective intervention can be severe in terms of human disease and death. There should be skepticism about adopting solutions that promise to permanently solve problems, not because such solutions cannot happen, but because of incomplete understanding of problems and proposed solutions, inadequate research into appropriate approaches to implementation, and the fact that the situation can change and new or old problems can emerge.

\subsection{ADAPTATION IS A PROCESS THAT REQUIRES SUSTAINED COMMITMENT}

Unwavering vigilance is required. It is easy for societies to become complacent and assume that problems are solved forever, and to not maintain efforts to monitor for the emergence or re-emergence of problems, or evaluate the ongoing effectiveness of solutions. Specific lessons from past experience suggest:

- Risks and their drivers change over time;

- A sustained commitment is required;

- Intervention on a small scale is warranted to test the effectiveness of a "solution"; and

- Monitoring and evaluation of interventions are important. 


\subsubsection{Risks and Their Drivers Change Over Time}

Effective public health management is committed to addressing changes in drivers or the disease itself promptly and efficiently. The burden of diseases such as malaria can increase with changes such as the development of drug-resistant parasites and pesticide-resistant vectors. Changes in land use, such as conversion of forest to rice cultivation, can create favorable conditions for disease vectors. Changing population demographics, particularly increasing numbers of individuals over age 65, suggest that the number of people sensitive to a particular health outcome (e.g., the elderly who are sensitive to heat stress) will increase, so that the potential vulnerability to the projected health impacts of climate change will increase (e.g., morbidity and mortality due to heat stress as the frequency and intensity of heat waves increase) (McMichael 2001). Whether or not the vulnerability of specific populations increases or decreases will depend on a variety of conditions, including socioeconomic factors, adaptive capacity, and the effectiveness of adaptive interventions.

Socioeconomic conditions can change, resulting in the easing, exacerbation, or creation of new health problems. For example, Weinstein and Woodward (in press) identify increasing social inequality as a possible cause of greater exposure to water-borne diseases. In contrast, improved social conditions can result in more favorable conditions to implementing actions to reduce a disease risk (Tol and Dowlatabadi 2002).

\subsubsection{A Sustained Commitment is Required}

The reemergence of yellow fever, dengue/dengue hemorrhagic fever, and malaria, particularly in the Americas, makes a strong case for the importance of a sustained commitment (Gubler and Wilson in press; Gubler and Kuno 1997; Gubler 1989). Mosquito control programs in the 1940s and 1950s resulted in effective control of the diseases in the Americas because mosquitoes carrying the diseases were controlled. This success unfortunately bred complacency and control measures were relaxed (and funds were diverted to other activities). In the 1960s and 1970s, these diseases reemerged.

Solid political will, adequate resources devoted to adaptation, and institutional commitments are needed to control health problems over the long term (Gubler and Wilson in press). One high-priority need is for adequate trained personnel and resources to be made available to countries where diseases are endemic and investments need to be maintained. The level of investment must address not only the burden of disease in that population but also the potential consequences if the disease moves across national boundaries (as was highlighted by the SARS outbreak of 2003). A related lesson is that many diseases cannot be eliminated, but they can be controlled (e.g., zoonotic diseases such as Lyme disease).

A strategy of eternal vigilance is a difficult one to follow. Once a problem is sufficiently reduced in scope that it fades from public consciousness, other problems emerge to take its place. We can assume that, in addition to having limited resources, there is a public attention budget that focuses on only a limited number of problems 
at one time. If a problem has insufficient immediate risk to keep it prominent in the public and political eye, other problems are likely to displace it and to attract scarce financial support.

\subsubsection{Intervention on a Small Scale is Warranted to Test} the Effectiveness of a "Solution"

An intervention, particularly something new, should be tested on a small scale. Testing can identify potential implementation problems or unintended consequences (e.g., for a new technology).

One example is the installation of tubewells in Bangladesh in an effort to provide a "safe" source of drinking water to a population that was experiencing high morbidity and mortality, especially among children, from water-related diarrheal diseases (e.g., cholera, dysentery, and other intestinal diseases) (Ebi et al. in press). However, in many regions of Bangladesh, the groundwater accessed by these wells has naturally occurring high concentrations of arsenic, a known carcinogen. The result has been called the largest mass poisoning of a population in history (Smith et al. 2000). Possibly 30 million out of the 125 million inhabitants of Bangladesh drink arsenic-contaminated water. The health consequences of exposure range from skin lesions to a variety of cancers. Because of the latency of arsenic-related cancers, morbidity and mortality from historical and current exposures may continue for approximately 20 years after exposures are discontinued. Testing of tubewells in Bangladesh might have identified the high arsenic levels in groundwater, in addition to the difficulties in getting people to appropriately use the wells. The lessons learned during the testing period can then be applied when an intervention is scaled up or deemed inappropriate or ineffective.

\subsubsection{Monitoring and Evaluation of Interventions are Important}

Policy makers should not be cavalier about the ease with which adaptation can be achieved, or the expected effectiveness of any policies implemented. We cannot assume that interventions will be effective and will continue to be so in perpetuity. Monitoring can identify where solutions have not worked and where further interventions are needed. Evaluation can identify why particular solutions did and did not work. Both monitoring and evaluation are needed to ensure interventions continue to have the intended effect and to identify unanticipated problems. Further, barriers to successful adaptation and the means of overcoming such barriers also need to be evaluated (Scheraga et al. 2003).

\subsection{AN UNDERSTANDING OF THE MULTIPLE AND INTERACTING DETERMINANTS OF DISEASE IS NEEDED TO INFORM THE DESIGN OF ADAPTATION STRATEGIES, POLICIES, AND MEASURES}

Climate change is only one of many factors influencing human health and social well-being; socioeconomic, political, and cultural considerations also influence 
vulnerability. The public health challenges presented by climate change need to be addressed within the context of other pressing issues, such as inadequate nutrition, access to clean water and sanitation, and other serious diseases such as HIV/AIDS and malaria.

Before designing and initiating an intervention, it is critical to gather information on the multiple and interacting determinants of disease, including both the immediate causes of the disease (e.g., a heatwave can increase morbidity and mortality) and other drivers associated with the disease (e.g., the land use change associated with increased urbanization can create an urban heat island that exacerbates the effect of a heat wave). It is important to understand contextual determinants of the disease, including the larger societal drivers such as population growth, poverty, and ecosystem changes.

Not surprisingly, poor health conditions and high vulnerability to disease within communities are often associated with, and caused by, poverty (WHO 2001). One of the outcomes of poverty (although perhaps also a cause) is inadequate public health infrastructure and access to health care. The consequence is a reduced ability to prevent and treat diseases, resulting in situations where controlling the spread of disease is difficult. Poverty is a factor increasing vulnerability to climate change across climate sensitive sectors. Substantially reducing or eliminating poverty would greatly enhance the ability of societies to cope with many aspects of climate variability and change, increase adaptive capacity, and perhaps dramatically reduce vulnerability. However, reducing poverty alone will not eliminate vulnerability; even wealthy countries with high adaptive capacity have pockets of poverty and groups of people who tend to be more vulnerable to climate variability and change, as demonstrated by the European heat wave in 2003 (Koppe et al. 2003) and the Chicago heatwave in 1995 (Scheraga and Grambsch 1998; NOAA 1995).

Climate is one of multiple factors that determine the range of a disease; changes in ecosystem can facilitate the emergence and re-emergence of disease, even under current climatic conditions (NAS 2001). Climate change can further change or disrupt natural systems, making it possible for diseases to spread or emerge in areas where they had been limited or had not existed, or for diseases to disappear by making areas less hospitable to the vector or the pathogen. For example, campylobacteriosis is an emerging human gastrointestinal disease, with a dramatic increase in cases in the last few decades. In New Zealand, natural vegetation has been replaced with pastoral farming (Weinstein and Woodward in press). This change in land use increased sources for disease (animal waste) and reduced the ability of natural vegetation to remove the wastes from runoff, resulting in contamination of half of New Zealand's rivers and streams. However, not all changes in land use have adverse consequences for human health; draining wetlands near human settlements can reduce habitats for disease-carrying mosquitoes. A challenge for decision-makers is to understand the multiple effects that any particular policy action may have, identify trade-offs that might have to be made (e.g., between protecting natural ecosystems and protecting human health), and then choose between the trade-offs. 
Part of the preparation to address the potential impacts of climate change is to improve our understanding of risks from the current climatic conditions that can become more frequent and/or extreme under climate change, such as those posed by heat waves and other extreme weather events, and to identify opportunities or positive effects that may occur (e.g., fewer deaths due to extreme cold). In addition, better understanding of potential risks from climate variability and change, and of the populations vulnerable to those risks, can help health authorities target surveillance, response, and other programs.

These lessons apply to sectors other than public health. For example, ecosystem changes may result in the spread of pests that can affect agricultural output. Using pesticides to control these pests can, in turn, increase the cost of agricultural production. And if climate change increases the frequency and intensity of rainfall events, there could be more runoff of pesticides from farms into rivers and streams, affecting water quality and wildlife. It is therefore critical that the effects of ecosystem changes are identified and that our understanding be improved of the mechanisms through which these changes occur.

\subsection{MULTIPLE POLITICAL, SOCIAL, ECONOMIC, TECHNOLOGICAL, AND HUMAN FACTORS DETERMINE WHETHER ADAPTATION STRATEGIES, POLICIES, AND MEASURES ARE EFFECTIVE}

Interventions need to be tailored to local circumstances. Differences in culture, education, knowledge, availability and affordability of technology, and other factors mean that a "one size fits all" approach is more likely to fail. Public health experience suggests the following lessons for the design of effective adaptation strategies, policies, and measures:

- It is important to understand the political, social, economic, and other forces that may affect implementation. Interventions are more effective if they are tailored to address local situations. Be wary of solutions that have not adequately accounted for all the factors that may affect implementation;

- Changing the behavior of individuals may be difficult; and

- Maladaptation and unintended consequences can occur in many different ways.

\subsubsection{It is Important to Understand the Political, Social, Economic, and Other Forces That may Affect Implementation. Interventions are More Effective If They are Tailored to Address Local Situations}

Scientific information is only one input into policy decisions. Selection and implementation of adaptation strategies, policies, and measures are done within a political, social, and economic context (Scheraga et al. 2003). Understanding this context, and perhaps modifying strategies to better fit into this context (if possible), can facilitate the acceptability of desirable adaptations.

Understanding the local situation is equally important for technological solutions. If the remedy is incompatible with local culture, it is unlikely to be adopted 
(Conde and Lonsdale 2005). Other factors also need to be considered, such as the local availability of resources; preferences that are a function of culture, traditions, religion, and other factors; the relative size of a vulnerable population (e.g., more elderly in one region than in another); equity considerations; and political feasibility. Different stakeholders have different, and perhaps competing, objectives, which can make the choice of specific adaptation measures difficult (Scheraga et al. 2003). This may mean more than simply consulting stakeholders, but empowering them to make decisions about appropriate responses.

This lesson is important for adaptation in all climate-sensitive sectors. Whether they are responding to sea level rise, change in crop yields, loss of biodiversity, or many of the other impacts of climate change, adaptation strategies that do not consider local circumstances can lead to lack of use, misapplication, and unintended consequences.

\subsubsection{Changing the Behavior of Individuals may be Difficult}

Adaptations that require individuals to change their behavior may be difficult to successfully implement. For example, convincing individuals to reduce their exposure to harmful UVB rays from the sun can be challenging if the change involves inconvenience or increased cost, although information campaigns to reduce UVB exposure have had some success (Lucas and McMichael in press). In addition, maladaptation could occur if the campaigns reduce sun exposure too much, limiting UVR exposure necessary to manufacture vitamin D.

The fact that individuals do not readily change their behavior is a challenge for all sectors. Successful campaigns to change individual behavior such as anti-smoking or anti-drunk driving efforts often started with strong stakeholder involvement (such as Mothers Against Drunk Driving), took years to succeed, and required not just public appeals but also incentives such as higher cigarette prices or stricter drunk driving penalties. This suggests that efforts to change individual behavior need to be undertaken, sometimes with incentives, in a sustained manner involving messages targeted to specific population subgroups. Better understanding of what has worked and why (and what hasn't worked and why not) can facilitate the development of more effective intervention programs.

\subsubsection{Maladaptation and Unintended Consequences can Occur in Many Different Ways}

Maladaptation occurs when current practices or behavior increase rather than decrease climate risks or risks from other stressors (Burton 1996). Maladaptation also occurs when interventions are implemented and scarce resources are used when adaptation was not necessary. And maladaptation can occur when a particular policy action reduces the risks posed by climate change, but has unintended consequences (e.g., a sea wall that protects infrastructure against rising sea levels, but leads to the destruction of coastal wetlands). Addressing maladaptation where it already has occurred can produce a "win-win" outcome: the interventions can 
address a situation that is a problem under current climate and can improve society's ability to adapt to future climate change (although additional adaptations may well be needed). Even if maladaptation is not occurring under current climate, it could arise over time with changing conditions. Systems that are well adapted now may become more vulnerable in the future as the climate changes. For this reason, ongoing monitoring and evaluation of implemented adaptation measures are needed.

\subsection{SURVEILLANCE AND EARLY WARNING SYSTEMS, COUPLED WITH EFFECTIVE RESPONSE CAPABILITIES, CAN REDUCE CURRENT AND FUTURE VULNERABILITY}

Surveillance is an important resource for identifying risks. Surveillance systems are designed to provide early intelligence on the emergence or re-emergence of health risks at specific locations in time for effective responses to be mounted (Wilson and Anker in press). However, to capitalize on this possibility, conventional surveillance systems need to account for and anticipate the potential effects of climate change. Surveillance systems will be needed in locations where changes in weather and climate may foster the spread of climate-sensitive diseases and vectors into new regions (NAS 2001). Similarly, information about the timing, location, and potential severity of possible changes in climate-sensitive sectors other than public health can be used to prioritize the placement of surveillance and early warning systems to alert populations to changing risk conditions (e.g., the spread of pests into agricultural areas and managed forests). Increased understanding is needed of how to design these systems where there is limited knowledge of the interactions of climate, ecosystems, and infectious diseases (NAS 2001).

Whereas surveillance systems are designed to detect and investigate adverse environmental conditions (such as high ambient air pollution concentrations) and disease outbreaks as they occur, early warning systems are designed to alert the population and relevant authorities that a disease outbreak is expected. Early warning systems can be very effective in preventing deaths, diseases, and injuries. The effectiveness of disease prediction depends on: an understanding of the mechanisms of disease transmission; reliable and up-to-date information on exposures and health outcomes; and a disease prediction model that is accurate, specific, and timely (Woodruff in press). Knowledge of disease transmission requires an understanding of the causes of the disease. Being able to predict disease outbreaks requires good historical data on which to base the prediction, as well as reliable and up-to-date monitoring data. The model needs to be sufficiently accurate and valid for the use to which it is put.

\subsubsection{Risk Prediction must be Coupled with Adequate Response Capabilities}

An early warning of a problem will be inadequate if it is not accompanied by an effective response capability, including a specific intervention plan. Once a warning is given, the public health system must have the capability to take effective measures 
to reduce the predicted risks. For example, the combination of a heat watch warning system and a response plan to reduce the exposure of vulnerable population groups to extreme heat led to a substantial reduction in deaths from extreme heat in Chicago in 1999 over that experienced in 1995 (Palecki et al. 2001).

\subsection{COLLABORATION AND COORDINATION OF RESEARCH AND RESPONSE ARE REQUIRED ACROSS SECTORS}

The effectiveness of strategies, policies, and measures for adaptation to climate variability and change in multiple sectors, including public health, are increasingly interdependent. If strong collaboration and coordination do not develop across different sectors and communities, then effective adaptation to climate change will be less likely, with needless human suffering. Consider, for example, farmers who respond to an influx of pests as climate changes by using more pesticides. This action may undermine the efforts of land-use managers who are constructing riparian buffer zones to reduce the run-off of pollution into rivers and streams that occurs with intense rainfall events.

Most science is currently conducted within narrow disciplines. Multidisciplinary approaches are required to understand the potential risks and benefits of climate change, and the possible response strategies to protect and enhance human health and well-being in the face of what the future will bring. Taking a more systemsbased approach allows the risks from climate change to be put into perspective with other drivers of disease, thus facilitating the identification of the most effective and efficient interventions (Martens et al. 2002).

\section{Implications for Public Health Policy}

The extent to which society is willing to expend resources to avoid the effects of climate change will depend in part on its perceptions of the risks posed by climate change, the perceived costs of the effort, ability to pay, and how much it is willing to risk possible negative consequences (NAS 1992; OTA 1993). Because resources need to be shared among a variety of public health problems, along with other problems of concern to society, the ideal situation is to direct resources to their highest valued uses to do the greatest public good (Scheraga et al. 2003). This, of course, is a social choice, not a scientific decision. Stakeholders may have conflicting desires, and conflict resolution is likely to be required. Policymakers will have to consider issues of equity (e.g., a decision that leads to differential health impacts among different demographic groups), efficiency (e.g., targeting those programs that will yield the greatest improvements to public health), and political feasibility. (For example, a policy that eliminates federal flood insurance to homeowners in high-risk coastal areas or flood plains may provide a strong incentive against building infrastructure in 'harms way,' but may not be politically feasible to implement.) Yet, failure to invest in adaptation may leave a nation poorly prepared 
to cope with adverse changes and increase the probability of severe consequences (Smit et al. 2001). Therefore, adaptation strategies, policies, and measures need to be considered as part of any larger policy portfolio.

Policy makers should not be cavalier about the ease with which adaptation can be achieved, or the expected effectiveness of any policies implemented. Not only must the potential health impacts of climate change and options for responding to these impacts be identified, but barriers to successful adaptation and the means of overcoming such barriers also need to be evaluated (Scheraga et al. 2003).

The development of adaptation strategies is complicated by the fact that significant uncertainties exist about the underlying science, the geographic and temporal scales of consequences, and the effectiveness of available response strategies (Smit et al. 2001). However, decisions must be made. The question is how best to make decisions despite the existence of uncertainties.

A major issue facing scientists and policymakers is how to communicate the scientific information that is available, the degree of certainty associated with this information, and the implications of uncertainties for the issues of concern to decision makers, resource managers, and other stakeholders. There are two dangers. One is that ineffective communication may result in the public taking little interest and not responding effectively. This is one reason why governments and intergovernmental agencies spend time and money to raise public awareness and to inform the public as to potential risks and benefits. The other danger is over-reaction. Once an issue (a risk or a threat) becomes the focus of public attention, there can be a high demand for information, reassurance, and action. Given the complexities and uncertainties associated with climate change, facilitating an informed and thoughtful public response could become as difficult as managing the risk itself.

Public health officials and researchers need to be educated to understand that climate is a risk factor for a range of diseases and that increasing resilience to current climate variability will sometimes be a step toward increasing adaptive capacity to future change. The climate is always changing, but it is projected to change at an increasingly rapid rate over the next few decades (Watson et al. 2001). This change may be accompanied by increasing climate variability, resulting in more frequent and more intense floods, droughts, heat waves, etc., that would strain the ability of public health systems worldwide to cope unless proactive policies are formulated and implemented.

Because both climate change and adaptation (whether reactive or anticipatory) to these changes are ongoing processes, active management of the risks and benefits of climate change needs to be mainstreamed into the design and implementation of disease control strategies and policies across the institutions and agencies responsible for maintaining and improving population health. The same is true for resource managers in other sectors. Some risk management activities will be extensions of current public health approaches that can be designed within existing operational responsibilities. For example, the design and implementation of early warning systems for climate-sensitive diseases might be a win-win strategy if the 
system both reduces the current burden of disease and increases adaptive capacity for future increases in disease incidence. However, one might choose not to invest in a specific adaptation to current climate variability if a cost-benefit analysis reveals that the net benefits of investing scare resources for prevention of other health risks is larger; such an analysis should consider the present value of the stream of net benefits over time to determine if an investment today is justified.

Other risk management activities will require modifications to current public health practices or systems. For example, a number of studies have suggested that the changing temperature and precipitation patterns projected to occur with climate change could increase the current range of epidemic malaria in Africa (e.g., Tanser et al. 2003; van Leishout et al. 2004; Ebi et al. in press). Development of closer collaboration between public health and meteorological agencies is needed in the placement of long-term monitoring stations to ensure that prevention activities are proactively implemented.

\section{Conclusions}

Better understanding is needed of the relationships between climate and health, and how these relationships could be effectively modified or influenced because the precise modifications needed to public health policies to address climate variability and change will depend on factors such as the health outcome; the expected severity of the climate impact; the location; the timing of when the impact is likely to occur; the effectiveness of measures in place to cope with the impact; other stressors that could increase or decrease resilience to impacts; the capacity of the population to cope with the impact; and who is expected to take action. In addition, to design effective adaptation options, research is needed to understand which public health interventions have and have not worked in the past, and why. Such research investments will provide the policy-relevant information needed by decision makers to formulate and implement appropriate response options. Resource managers and decision-makers outside the public health community are faced with similar issues.

Policy makers dealing with multiple social objectives (e.g., elimination of poverty, support for agriculture, promotion of economic growth, protection of cultural resources) and competing stakeholder desires must make difficult choices as they allocate scarce human and financial resources (Scheraga et al. 2003). For this reason, the IPCC suggested that it is useful to view climate change as part of the larger challenge of sustainable development (Smit et al. 2001). Climate policies - including those intended to protect public health - can be more effective when consistently embedded within broader strategies designed to address national and regional development pathways. The impact of climate variability and change, climate policy responses, and associated socioeconomic development will affect the ability of communities to achieve development goals.

Despite difficulties and uncertainties, adaptation is critical to prepare individuals, communities, governments, and international agencies for the changes that are 
coming. Coping with the potential effects of climate variability and change will be a complex and ongoing process requiring action at all levels. Understanding the lessons learned from public health can facilitate the process of designing and implementing strategies, policies, and measures to reduce vulnerability and increase adaptive capacity.

\section{Acknowledgments}

Financial support was provided by the Electric Power Research Institute and the U.S. Environmental Protection Agency's Office of Research and Development, specifically its Global Change Research Program and the National Center for Environmental Assessment.

\section{References}

Burton, I.: 1996, 'The growth of adaptation capacity: Practice and policy', in J.B. Smith, N. Bhatti, G. Menzhulin, R. Benioff, M. Budyko, M. Campos, B. Jallow and F. Rijsberman (eds.), Adapting to Climate Change, New York, Springer, pp. 55-67.

Conde, C. and Lonsdale, K.: 2005, 'Engaging stakeholders in the adaptation process', in B. Lim, E. Spanger-Siegfried, I. Burton, E. Malone S. Huq (eds.), Adaptation Policy Frameworks for Climate Change, Cambridge, Cambridge University Press, pp. 47-66.

Gubler, D.: 1997, 'Dengue and dengue hemorrhagic fever: Its history and resurgence as a global public health problem', in D. Gubler and G. Kuno (eds.), Dengue and Dengue Hemorrhagic Fever, New York, CAB International, pp. 1-22.

Gubler, D.J.: 1989, 'Aedes aegypti and Aedes aegypti-borne disease control in the 1990s: Top down and bottom up', American Journal of Tropical Medicine and Hygiene 40, 571-578.

Koppe, C., Jendritzky, G., Kovats, R.S. and Menne, B.: 2003, Heatwaves: Impacts and Responses, Copenhagen: World Health Organization, 2003.

Marten, P., Rotmans, J. and Rothman, D.S.: 2002, 'Integrated assessment modeling of human health impacts', in P. Martens and A.J. McMichael (eds.), Environmental Change, Climate and Health, London, Cambridge University Press, pp. 197-225.

McMichael, A.J., Githeko, A., Akhtar, R., Carcavallo, R., Gubler, G., Haines, A., et al.: 2001, 'Human health', in J.J. McCarthy, O.F. Canziani, N.A. Leary, D.J. Dokken and K.S. White (eds.), The Third Assessment Report of the Intergovernmental Panel on Climate Change, Cambridge, Cambridge University, pp. 451-486.

McMichael, T.: 2001, Human Frontiers, Environments and Disease: Past Patterns, Uncertain Futures, London, Cambridge University Press.

NAS: 1992, Policy Implications of Greenhouse Warming: Mitigation, Adaptation, and the Science Base, National Academy of Sciences, National Academy Press, Washington, DC.

NAS Committee on Climate Ecosystems Infectious Disease and Human Health Board on Atmospheric Sciences and Climate and National Research Council: 2001, Under the Weather: Climate, Ecosystems, and Infectious Disease, Washington, DC: National Academics Press.

NOAA (National Oceanic and Atmospheric Administration): 1995, July 1995 Heat Wave, Natural Disaster Survey Report, Washington, DC: United States Department of Commerce.

OTA: 1993, Preparing for an Uncertain Climate, Office of Technology Assessment, U.S. Government Printing Office, Washington, DC. 
Palecki, M.A., Changnon, S.A. and Kunkel, K.E.: 2001, 'The nature and impacts of the July 1999 heat wave in the Midwestern United States: Learning from the lessons of 1995', Bulletin of the American Meterological Society 82, 1353-1367.

Scheraga, J.D. and Grambsch, A.E.: 1998, 'Risks, opportunities, and adaptation to climate change', Climate Research 10, 85-95.

Scheraga, J.D. and Smith, A.E.: 1990, 'Environmental policy assessment in the 1990s', Forum Soc. Econ. 20, 33-39.

Scheraga, J.D., Ebi, K.L., Furlow, J. and Moreno, A.R.: 2003, 'From science to policy: Developing responses to climate change', in A.J. McMichael, D. Campbell-Lendrum, C.F. Corvalan, K.L. Ebi, A. Githeko, J.D. Scheraga and A. Woodward (eds.), Climate Change and Human Health: Risks and Responses, WHO/WMO/UNEP, pp. 237-266.

Smit, B., Pilifosova, O., Burton, I., Challenger, B., Huq, S., Klein, R. and Yohe, G.: 2001, 'Adaptation to climate change in the context of sustainable development and equity', in J. McCarthy, O. Canziana, N. Leary, D. Dokken and K. White (eds.), Climate Change 2001: Impacts, Adaptation, and Vulnerability, New York, Cambridge University Press, pp. 877-912.

Smith, A.H., Lingas, E.O. and Rahman, M.: 2000, 'Contamination of drinking-water by arsenic in Bangladesh: A public health emergency', Bull World Health Organ 78, 1093-1103.

Smith, J.B. and Lenhart, S.S.: 1996, 'Climate change adaptation policy options', Climate Research 6, 193-201.

Tanser, F.C., Sharp, B. and leSueur, D.: 2003, 'Potential effect of climate change on malaria transmission in Africa', Lancet 362, 1792-1798.

Tol, R.S.J. and Dowlatabadi, H.: 2002, 'Vector-borne diseases, development, and climate change', Integrated Environmental Assessment 2, 173-181.

Van Lieshout, M., Kovats, R.S., Livermore, M.T.J. and Martens, P.: 2004, 'Climate change and malaria: Analysis of the SRES climate and socio-economic scenarios', Global Environmental Change 14, 87-99.

Watson, R.T., Albritton, D.L., Barker, T., Bashmakov, I.A., Canziani, O., Christ, R., et al.: 2001, 'Climate Change 2001: Synthesis Report. A Report of the Intergovernmental Panel on Climate Change', IPCC Secretariat c/o World Meteorological Organization, Geneva, Switzerland.

World Health Organization: 2001, 'Macroeconomics and health: Investing in health for economic development. Report of the Commission on Macroeconomics and Health', Geneva, Switzerland. 